\title{
El criterio de causalidad en la valoración de la imputabilidad de los trastornos de la personalidad.
}

\author{
The criterion of causality in the assessment of the \\ imputability of personality disorders.
}

\section{A. Villarejo Ramos ${ }^{1}$}

\section{RESUMEN}

La determinación de la imputabilidad penal de un sujeto con trastorno de personalidad que ha cometido un delito se basa en la comprobación de cuatro criterios: el cualitativo, el cuantitativo, el cronológico y el de causalidad. Este último es el objeto de nuestro trabajo.

Establecer la causalidad implica achacar al trastorno de personalidad la comisión de la conducta antijurídica, ejercicio no exento de dificultades. En primer lugar, y desde una perspectiva filosófica, porque supone una tarea de observación eminentemente empírica y probabilística. En segundo lugar, desde la óptica psicológica, porque la causalidad de la conducta no radica exclusivamente en el sujeto que la realiza, sino en el juego de interacción de éste con la constelación de estímulos ambientales que actúan sobre él, lo que implica la investigación de tales factores situacionales y la forma en que éstos "intervienen" sobre la persona. Y finalmente, desde el punto de vista psiquiátrico-forense, hemos de diagnosticar los rasgos centrales de personalidad que presenta el infractor y que son afectados por determinadas situaciones ambientales y comprobar que las conductas imputadas se ajustan en su patoplastia a las consistentes respuestas comportamentales esperadas según dicha interacción.

En otras palabras, para determinar la causalidad entre un acto delictivo y el trastorno de personalidad de quien lo comete, hemos de estudiar la interacción ambiente/rasgos centrales de personalidad y comprobar que el hecho imputable responde al patrón de comportamiento habitual en esa determinada personalidad.

Palabras clave: Trastornos de personalidad. Imputabilidad. Relación de causalidad. Rasgos centrales. Comportamientos consistentes. Psiquiatría forense.

\section{ABSTRACT}

The determination of the penal imputability of an individual with a personality disorder who has committed an offence is based on the examination of four factors: qualitative, quantitative, and chronological, as well as the criterion of causality. The last is the object of our work.

Establishing causality implies attributing personality traits to the commission of unlawful behaviour, an exercise not free of difficulties. Firstly, from a philosophical perspective, because it supposes a task of observation eminently empirical and probabilist. Secondly, from the psychological perspective, because the causality of the behaviour isn't rooted exclusively in the individual who performs it, but in the interaction between that individual and the environmental stimuli that are acting upon him, which implies the investigation of such environmental factors and of the way they influence the individual. And finally, from the forensic-psychiatric point of view, we have to diagnose the nuclear personality traits that the infractor demonstrates, which are affected by definite environmental situations, and verify that the imputed behaviour fits in its patoplasty with the consistent behavioural answers expected according to said interaction.

In other words, to determine the causality between an unlawful act and the personality trait of the one who commits it, we have to study the interaction environment/nuclear traits of personality and verify that the imputable fact answers to the pattern of habitual behaviour in that particular personality.

Key words: Personality disorders. Imputability. Relation of causality. Nuclear traits. Consistent behaviour. Forensic psychiatry.

Correspondencia: Alberto Villarejo Ramos. Clínica Médico Forense de Cádiz. C/ Los Balbos s/n. Tlfno: 956013060.

${ }^{1}$ Médico Forense. Especialista en Medicina Legal y Forense. Profesor Asociado de la Cátedra de Medicina Legal de la Universidad de Cádiz. Colaborador en el Departamento de Neurociencias de la Universidad de Cádiz. 


\section{INTRODUCCIÓN.}

La jurisprudencia española, para determinar la imputabilidad de una persona con alteraciones o anomalías psíquicas en relación a la comisión de un comportamiento antijurídico, ha venido considerando tradicionalmente como criterios de valoración los de cualidad, cantidad, cronológico y de causalidad. En un trabajo anterior nuestro [I], exponíamos los criterios de cantidad y cualidad relacionados con los sujetos con trastorno de personalidad y hacíamos referencia a aspectos personológicos que, en nuestra opinión, deberían ser considerados, mas allá de los meros trastornos intelectuales o volitivos para valorar la imputabilidad de estas personas.

En el presente trabajo estudiaremos el criterio de causalidad. Si entendemos la causalidad según la Real Academia de la Lengua Española como "causa, origen, principio" o como "ley en virtud de la cual se producen efectos" encontraremos grandes escollos si, atendiendo a nuestro actual Código Penal, pretendemos aplicar tal término a los comportamientos antijurídicos perpetrados por sujetos con trastornos de la personalidad. En efecto, pues los trastornos de la personalidad "per se" no pueden considerarse causa de ninguna conducta, legal o ilegal, si no es dentro de un determinado contexto situacional. Por tanto, el criterio de causalidad debe basarse en la interacción de los factores situacionales con la estructura de la personalidad del imputado. Trataremos de llegar a esta conclusión a través de una crítica del concepto jurídico de causalidad desde una, forzosamente breve, perspectiva filosófica y desde las ópticas psicológica y psiquiátrico-forense.

\section{CONCEPTO DE CAUSALIDAD.}

\section{EL PUNTO DE VISTA JURÍDICO:}

El artículo 20 del vigente Código Penal Español de 1995 contempla que: "Están exentos de responsabilidad criminal: $I^{\circ}$. El que al tiempo de cometer la infracción penal, a causa de cualquier anomalía o alteración psíquica, no pueda comprender la ilicitud del hecho o actuar conforme a esa comprensión."

La Jurisprudencia española, por otra parte, ha reconocido en retiradas ocasiones que los requisitos para aplicar tal eximente están basados en una cuádruple comprobación (Carrasco, 1997 [2]: I. La naturaleza de la perturbación (criterio cualitativo). 2. La intensidad y grado de la perturbación (criterio cuantitativo). 3. Duración del trastorno y permanencia del mismo (criterio cronológico), si bien el propio autor reconoce como irrelevantes estos aspectos temporales, concediendo mas importancia a la concurrencia de la alteración psicopatológica con la acción delictiva, pues así lo exige el propio precepto, donde se lee “... al tiempo de cometer la infracción penal” y 4. Relación de causalidad o de sentido entre el trastorno psíquico y el hecho delictivo, siendo este último criterio el objeto de nuestro estudio.

Desde la perspectiva del criterio de causalidad, el artículo 20 referido implica inimputabilidad cuando:

I. En una persona se da una CAUSA, "cualquier anomalía o alteración psíquica" (el trastorno de la personalidad en nuestro caso) y

2. Que por tal causa se origine un determinado EFECTO, cometer una infracción penal.

Es decir, la ley penal exige para aplicar esta eximente que el trastorno de la personalidad deba ser causa de la infracción penal. Así lo recoge el magistrado Soto Nieto (citado por Martínez, López y Díaz [3]) que asegura que "...ha de tenerse en cuenta que el tipo de delito ha de estar en relación con el tipo de psicopatía para que modifique la imputabilidad.... La anormalidad caracterológica del psicópata ha de estar en relación causal con el hecho delictivo..." 
No obstante, podemos encontrar dificultades a la hora de determinar este nexo causal, tanto desde un punto de vista filosófico, como psicológico y psiquiátrico-forense.

\section{EL PUNTO DE VISTA FILOSÓFICO :}

Hume ya explicó en el siglo XVIII que la creencia de que ciertos acontecimientos precedieran a otros en el tiempo, es decir, que aquellos fueran la causa de éstos no estaba fundamentada en ninguna razón lógica. El filosofo escocés aseguraba que si tal relación causal había ocurrido en el pasado, no tendría porque darse nuevamente en el futuro, sino que atribuimos tal causalidad a una observación eminentemente empírica.

Por otro lado, la filosofía nos enseña igualmente que no hay relación lineal entre causa y efecto, pues una sola causa pude ocasionar varios efectos o un solo efecto puede estar originado por varias causas distintas. Distingue, así, tres tipos de causas:

- Causa necesaria. Es un acontecimiento que debe preceder a otro para que este último ocurra. Una causa necesaria no implica que sea suficiente.

- Causa suficiente. Es aquella que es adecuada en sí misma para causar el efecto. No es necesario que otro factor se asocie a ella.

- Causas contribuyentes. Son factores que aumentan la posibilidad de que se produzca un efecto.

Atendiendo a esto, el trastorno de personalidad, por sí mismo no es causa necesaria de ninguna infracción penal, puesto que esta puede ser cometida en ausencia de aquel. Tampoco el trastorno de la personalidad es causa suficiente para cometer actos antijurídicos, pues no todos los sujetos con trastorno de la personalidad son autores de ellos. Finalmente, creemos que los trastornos de la personalidad si pueden ser considerados como causas contribuyentes, ya que, como veremos luego, algunos trastornos de la personalidad pueden aumentar la probabilidad de que se produzca un delito.

Si consideramos a los trastornos de la personalidad como causa contribuyente, icuáles son las otras causas que junto a ellos producen el efecto de la conducta antijurídica?

\section{EL PUNTO DE VISTA PSICOLÓGICO.}

La comisión de una infracción penal es, en términos psicológicos, una conducta y según Mira López, citado por Marcó, Martí y Pons en 1990 [4] los mismos mecanismos psicológicos intervienen en la ejecución de los actos legales que en la de los actos ilegales. Nos interesa pues, estudiar las causa generales de la conducta.

Según la teoría de las "causas internas", citada por Labrador [5], la causa de la conducta estaría dentro de las personas, en las que existe "algo" ("mente o "carácter") que es el responsable de nuestras conductas. Atendiendo a esta teoría, podríamos considerar que la personalidad o el trastorno de la personalidad por si mismo, explicaría la conducta de estos sujetos, sería su causa.

No obstante, algunos autores, entre ellos el mismo Labrador, critican la teoría de las causas internas y mantiene que son las "causas externas" las verdaderas motivaciones de la conducta. Según Labrador, las condiciones o incitaciones del medio controlan más las conductas humanas que ese "algo" interno. Para apoyar su teoría propone el siguiente ejemplo: Si un niño estudia es porque es responsable, si no es porque es un indolente; pero si al niño que estudia le facilitamos acudir a una fiesta o a una película, dejará de ser responsable y se convertirá en indolente; y si al indolente le ofrecemos un premio importante por estudiar, dejará de ser indolente y se convertirá en responsable. 
Según lo expuesto anteriormente, la personalidad, o el trastorno de la personalidad, no explicaría la conducta, no sería la causa de ella. Labrador ve, por el contrario, necesario buscar las explicaciones de la conducta en las características del ambiente ("causas externas"), tanto actuales como anteriores, que actúan como estímulos y condicionan la respuesta del individuo.

Ya parecen superadas, no obstante, las antiguas disputas psicológicas entre las causas internas y las causas externas de la conducta, entre rasguismo y ambientalismo. La teoría interaccionista, considera, por el contrario, que los estímulos ambientales actúan sobre determinada personalidad, la cual los modula y matiza, dándoles distintos significados y confiriendo determinada plasticidad a las respuestas.

Eysenck ( 1977) [6] propone que la criminalidad es el producto de la influencia de variables ambientales sobre individuos con determinadas predisposiciones genéticas y el aprendizaje por condicionamiento clásico. Es decir, que una conducta criminal se llevará o no a cabo atendiendo a las dimensiones de la personalidad (para el autor, neuroticismo, extroversión y psicoticismo), los estímulos ambientales y las respuestas que el entorno de a las propias conductas de la persona.

\section{EL PUNTO DE VISTA PSIQUIÁTRICO-FORENSE:}

El criterio jurídico de causalidad nos exige determinar si la conducta antinormativa es consecuencia del trastorno de la personalidad, es decir, si tal conducta es un "síntoma" de determinado trastorno de personalidad.

En ciertos trastornos mentales este nexo causal parece evidente. Así, por ejemplo, resulta fácil hallar relación de causalidad entre un homicidio perpetrado por un esquizofrénico paranoide y su creencia delirante de ser atacado y sus alucinaciones auditivas de tipo imperativo. Existe en este caso, podríamos decir, una "causa interna", representada por los síntomas psicóticos.

También es fácil determinar el nexo causal entre una agresión sexual cometida por un deficiente mental y el déficit intelectual que padece o su impulsividad sexual. Encontramos igualmente en este caso unas "causas internas".

Sin embargo, tales causas o síntomas internos no aparecen en sujetos con trastorno de personalidad, pues no sufren delirios o alucinaciones, ni padecen deficiencia intelectual.

Para explicar la causalidad de una conducta delictiva desde el punto de vista de la psiquiatría forense, estamos obligados a descubrir los estímulos del ambiente que actúan sobre la personalidad y obligados, por otra parte, a comprender como tales estímulos interaccionan con las características particulares del sujeto y cuya interacción determina una acción antijurídica determinada.

Trataremos de probar que existe una causalidad psiquiátrico-forense entre los individuos con trastorno de personalidad y sus eventuales comportamientos delictivos mas allá de la presencia o no de "síntomas internos".

\section{ANÁLISIS PSIQUIÁTRICO-FORENSE DEL CRITERIO DE CAUSALIDAD.} INTERACCIÓN CAUSAL.

Como hemos visto arriba, en relación con los trastornos de la personalidad no podemos esperar establecer una relación neta entre las "alteraciones o anomalías psíquicas" de las que habla el Código penal español y la conducta delictiva. Para encontrar las causas que origina un comportamiento antijurídico en un sujeto con trastorno de personalidad hemos de estudiar, como hemos expuesto ya, el juego de interacción entre los estímulos ambientales y la propia estructura de la personalidad.

En este sentido se expresa Mira y López, citado por Marcó, Martí y Pons (1990) [7], que propone la existencia de motivaciones delictivas endógenas (inherentes a la personalidad) y motivaciones exógenas (provenientes del ambiente) que se combinan en variable proporción. Skrapec 
(2000) [8] cita a Raine quien habla del síndrome de criminalidad como el producto de unas predisposiciones biológicas (incluidas las genéticas) para, por ejemplo, cometer acciones de tipo impulsivo y violento, y de sus interacciones con factores psicológicos y sociales.

Carrasco Gómez [9] afirma que el estudio de las personalidades anormales con conductas antisociales no es tan solo un problema de personalidad, sino que la persona es el resultado de su interacción con el medio ambiente y añade que si se buscan modificaciones de la imputabilidad en (estos) casos de personalidades antisociales, tendría que ser con base en la consideración de factores sociales y no recurriendo, únicamente, al desequilibrio psíquico bajo la óptica y criterios del libre albedrío.

Para Esbec y Delgado Bueno [ 10 ] la conducta no solo se explica en función de la persona (rasguismo) ni solo en función de la situación puntual (situacionismo), sino en función de la interacción entre ambos factores (interaccionismo) dentro de un contexto sociocultural determinado y citan a Debuyst et al (1990) que consideran que "al margen del carácter patológico que pueda existir, es el interior de una interrelación sociedad/delincuente en donde puede situarse el problema".

El penalista Muñoz Conde afirmaba ya en 1982 [I I ], en relación a la imputabilidad penal que "es muy probable que si no en su totalidad, si en gran parte, los actos humanos, sean mas producto de la estructura social, de las características sociales, culturales y antropológicas de cada individuo, de su ignorada constitución biológica a los niveles mas íntimos, de sus creencias religiosas, de su educación, profesión, trabajo, etc... que eso que ampulosamente se llama libertad."

Es decir, las causas de un delito cometido por un sujeto con trastorno de personalidad deben ser buscadas en la interacción de la estructura de personalidad del sujeto con los múltiples factores ambientales que le rodean.

Por otra parte, Millon [1 2] hace referencia a los enfoques contemporáneos y del desarrollo para explicar el comportamiento del individuo. El primero hace un análisis transversal de los acontecimientos actuales, es decir, el estado del individuo y su entorno tal y como existe en el presente; y el segundo, trata de explicar la conducta del sujeto en base a las circunstancias aparecidas en el pasado de este.

\section{LA CAUSALIDAD COMO PROBABILIDAD.}

Este epígrafe trata de responder a la pregunta:itodas las conductas anómalas son consecuencias de una personalidad anómala?, o, más correctamente y desde la perspectiva interaccionista, isiempre que existe un delito es consecuencia de la interacción estímulo ambiental/trastorno de la personalidad?; es decir, ien qué medida se puede establecer la causalidad entre esta interacción y la conducta criminal?

Ortega-Monasterio (199|) [13] afirma que el criterio de causalidad (para establecer la imputabilidad en cualquier trastorno psíquico) supone que sin la existencia del trastorno no se hubiera realizado determinada conducta antinormativa y objeta que con ello, en realidad, tal relación de causaefecto se trata de una suposición, dado que no se sabe si se hubiese realizado la conducta imputable en ausencia del trastorno. Esta relación es más un cierto grado de probabilidad que una causalidad en el sentido exacto de las ciencias naturales (Dalgardo, 1986, citado por Ortega-Monasterio).

Tal aserto puede ser aplicado en el caso de los trastornos de la personalidad. Sin embargo, la observación empírica y algunas investigaciones parecen poner de manifiesto que existe una mayor probabilidad de que se sucedan ciertos comportamientos delictivos asociados con determinados trastornos de la personalidad. Así, por ejemplo, en relación a los trastornos antisociales, Raine y Sanmartín, (2000) [ 14 ] informan que en los Estados Unidos, aunque se estima que estos sujetos son solo un $1 \%$ de la población total, constituyen, en cambio, el 25\% de la población reclusa. Para Hare 
(2000) [ 15] queda claro que un psicópata, por los rasgos de su personalidad, tiene muchas más probabilidades que el resto de los individuos de transgredir las reglas y las leyes de la sociedad. Esta asociación entre psicopatía y crimen se verifica, según el autor mediante la aplicación de la escala PCLR (Psychopathy Checklist Revised).

La respuesta, pues, es que, aunque no siempre ha de ser así, existe una alta probabilidad de que, al menos en relación al trastorno antisocial, si lo sea. Es decir, que las causas del delito muy probablemente, radiquen en tal interacción.

\section{LA CAUSALIDAD BASADA EN LA CONSISTENCIA DE LA CONDUCTA Y EN LA CENTRALIDAD DE LOS RASGOS DE LA PERSONALIDAD.}

Establecer la causalidad también nos lleva a preguntarnos iTodas las conductas de una personalidad anómala son anómalas? es decir, isiempre que existe un trastorno de personalidad debemos esperar una conducta anormal?

En un principio cabe hablar de que todas las conductas de un determinado sujeto son debidas a su personalidad, pues la personalidad es el patrón estable de pensamientos, afectos y comportamientos. El trastorno de la personalidad sería, según DSM IV, [ I6] un patrón permanente e inflexible de experiencia interna y de comportamiento, que se aparta acusadamente de las expectativas de la cultura del sujeto, tiene su inicio en la adolescencia o principio de la edad adulta, es estable a lo largo del tiempo y comporta malestar o perjuicios para el sujeto.

Según tales definiciones, el comportamiento de un sujeto con trastorno de personalidad siempre ha de ser anormal, pues dicho comportamiento se caracteriza por su estabilidad y permanencia; de hecho, es el propio patrón de conducta el que nos permite, entre otros elementos, establecer el diagnóstico de trastorno de la personalidad.

No obstante, Mischel, citado por Millon, (2000) [17] demostró en 1969 que los comportamientos estaban afectados significativamente por las variaciones situacionales, como hemos venido desarrollando. Según esto, el propio concepto de personalidad se viene abajo (Millon, 2000) [18].

Sin embargo, el mismo Mischel (1979) [19] afirma que las personas se comportan de una forma estable en ciertas situaciones y variable en otras. Esto es debido a que cada individuo posee un pequeño grupo de rasgos que persisten en el tiempo y en distintas situaciones (rasgos estables) y manifiestan comportamientos similares ante determinados estímulos ambientales (comportamientos consistentes), pero también posee otros rasgos que no son tan estables y que permiten la aparición de conductas diversas en función de la situación que las origina.

Con independencia de la validez absoluta del planteamiento de Mischel, fundamentalmente situacionista, Millon [20] observa que cada individuo posee un pequeño grupo de rasgos primarios o centrales que persisten en el tiempo y en distintas situaciones y muestran un alto grado de consistencia y estabilidad. A ellos es a los que se refiere el autor cuando habla de personalidad. Catell, (citado por Thetford y Walsh) [2I] por su parte, distingue entre los rasgos superficiales y los rasgos esenciales. Los rasgos esenciales son definidos como "factores estructurales simples" y representan la fuente subyacente que constituye una dimensión independiente unitaria y un elemento constructivo de la personalidad y que parecen explicar la mayor parte de las conductas. Propone 16 factores dicotómicos para la descripción de la personalidad. Los rasgos superficiales son simples conjuntos conductuales que tienden a aparecer y desaparecer conjuntamente.

Eysenck y Eysenck ( 1985 ) (Marcó, Martí y Pons)[22] consideran que los rasgos son esencialmente factores disposicionales que determinan regular y persistentemente nuestra conducta en tipos de situaciones diferentes, es decir, representan la conducta habitual de un individuo, mientras que el "estado" pondría de manifiesto su conducta atípica. 
Como vemos, en cada sujeto podemos encontrar unos rasgos de la personalidad, llamémosles primarios, centrales, esenciales o disposicionales, que condicionan permanentemente la conducta del individuo, pero también otras características personales menos estructuradas que serían responsables de comportamientos atípicos y no persistentes.

Por tanto, la respuesta a las cuestiones planteadas arriba sería "no", pues la conducta anómala debe estar relacionada con los rasgos centrales o esenciales de la personalidad, lo cual explica dos cosas:

En primer lugar, que no todas las situaciones producen la misma respuesta (conducta) en un determinado tipo de personalidad, pues algunas situaciones pueden "afectar" a rasgos centrales de una personalidad y producir una conducta "específica" y otras situaciones solo afectan a características no esenciales de la personalidad y producir conductas no específicas.

Pensemos en un sujeto narcisista ante el abandono de su esposa. Esta situación afecta y lesiona su orgullo y la imagen admirable que tiene de si mismo, de forma que dará una respuesta según su habitual actitud arrogante y comportamiento interpersonal explotador, marcada por hostilidad y agresividad hacia ella. Esta respuesta se repetirá casi invariablemente siempre que se den situaciones similares, pues dichos estímulos situacionales afectan a los rasgos centrales de la personalidad narcisista que hemos descrito.

Sin embargo, este sujeto ante cualquier otra situación, alguien que le saluda en la calle, por ejemplo, puede reaccionar de formas muy distintas ya que este estímulo no tiene porque afectar a rasgos primarios de su personalidad. La respuesta puede ser incluso normal o socialmente adaptada como haría cualquier otro sujeto sin trastorno de la personalidad.

Estas diferentes respuestas (conductas) en los trastornos de la personalidad son explicadas por Irastorza [23] proponiendo la teoría del pluralismo mental. Según esta teoría, en los trastornos de la personalidad existe en el mismo sujeto una convivencia de mentes normales y desviadas que actúan con relativa independencia y producen comportamientos distintos, según la mente que esté "activada". El sujeto se convertiría "en lo que sea la mente activada" Ello explicaría, según el autor, que personas con un comportamiento normal en la sociedad, en la familia o en el trabajo, sean psicópatas criminales, violadores, etc, pues tales sujetos pueden poseer al mismo tiempo una mente asesina y una mente normal.

La consistencia de la conducta y la existencia de rasgos primarios o centrales explican, por otra parte, que una misma situación produzca respuestas distintas en personalidades distintas al afectar a los respectivos rasgos primarios, como es lógico. Un paranoide no reaccionaría de la misma forma que un histriónico ante alguien que le apoya la mano en el hombro.

\section{CONCLUSIÓN.}

En primer lugar diremos que determinar la causalidad entre un hecho delictivo y el trastorno de personalidad padecido por quien lo cometió es una tarea fuera del método científico y sujeta más bien a la observación empírica. Y esto es así porque, si bien es cierto que el sujeto en cuestión ha podido presentar un patrón determinado de comportamiento en el pasado, nada nos asegura que este patrón sea el responsable de la conducta que se imputa en el presente. Por otro lado, establecer la causalidad entre una conducta ilegal y el trastorno de personalidad del infractor es responder a la cuestión de si este hubiese cometido el mismo delito en el caso de no padecer dicha alteración, es decir, es movernos en el terreno meramente probabilístico.

Si bien es cierto que la discusión sobre ambas aseveraciones es estéril; la primera, porque no nos queda mas remedio que admitir como valida esa observación empírica; y la segunda porque es imposible demostrar lo contrario. 
En segundo lugar, las causas de una conducta, sea esta legal o no, han de ser buscadas en un conjunto de factores fundamentados en la interacción de los elementos ambientales presentes o pasados con la estructura de la personalidad del imputado. Encontrar esos estímulos situacionales y relacionarlos con la personalidad del delincuente es imprescindible para comprender la psicogénesis del delito y establecer la relación de causalidad entre este y el trastorno de personalidad.

En tercer lugar, y en virtud de la consistencia de ciertas conductas y la especificidad de determinados rasgos (rasgos primarios, centrales o esenciales) que caracterizan a la personalidad, podemos establecer el nexo de causalidad entre una conducta antinormativa y el trastorno de personalidad del imputado, cuando tal conducta es la consecuencia de la interacción de uno o varios estímulos ambientales específicos con un rasgo primario o central de dicha personalidad (fig. I). En caso contrario, es decir, cuando un estímulo situacional no específico afecta a una característica no central o esencial de la personalidad, se producirá una conducta inespecífica, que podría presentarse en cualquier tipo de personalidad, lo que nos impide establecer nexo causal entre la acción ilegal y el trastorno de personalidad (fig. 2). En cierta manera se puede explicar esta relación causal mediante una teoría de llave-cerradura.

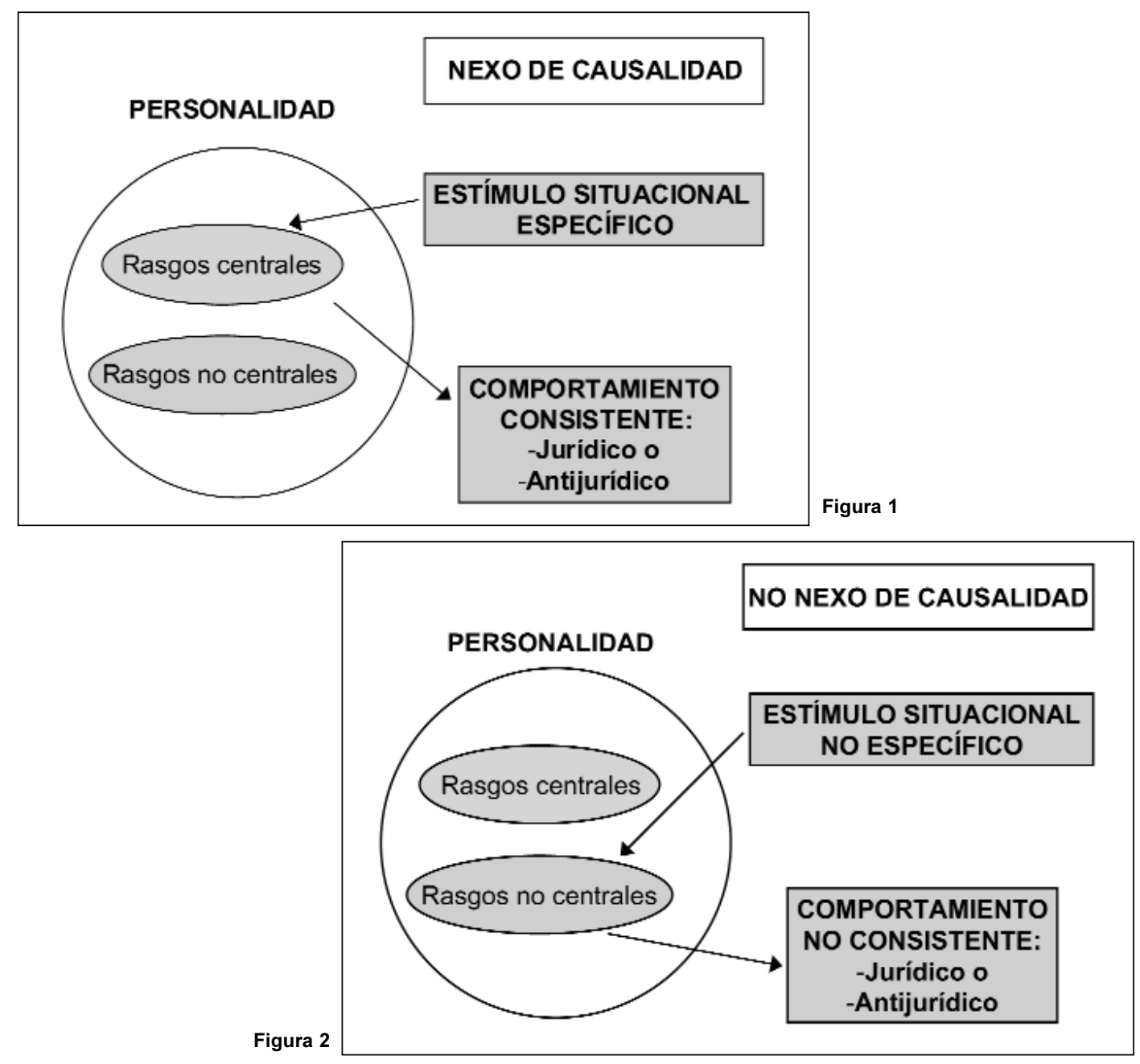


Las anteriores consideraciones, finalmente, nos exige investigar el tipo de estímulos situacionales tanto actuales como anteriores que desencadena la conducta antijurídica y relacionarlo con los rasgos centrales de la personalidad para poder establecer el nexo causal entre estos y el delito imputado.

\section{BIBILIOGRAFÍA:}

I. Villarejo Ramos A: Reflexión sobre la imputabilidad de los trastornos de la personalidad. Cuadernos de Medicina Forense. 2001;24:2530 .

2. Carrasco Gómez J]; Maza Martín JM: Manual de Psiquiatría Legal y Forense. Ed. La Ley-Actualidad. Madrid, 1997. II.5-14.

3. Martínez Díaz T; López Blanco F; Díaz Fernández ML: Los trastornos de la personalidad en el derecho penal: Estudio de casos del Tribunal Supremo. Psicopatología clínica, legal y forense. 2001; I, I:87-101.

4. Marcó Ribé J; Martí Tusquets JL; Pons Bartran R: Psiquiatría Forense. Ed. Salvat. Barcelona, 1990. 39.

5. Labrador F): El control de las conductas humanas. iPor qué funcionan las conductas?. Cuadernos de Derecho Judicial. 1994; XIX:1923.

6. Eysenck HJ: Crime and Personality. Ed. Paladin ( $3^{\mathrm{a}}$ ed.) London, 1997.

7. Marcó Ribé J; Martí Tusquets JL; Pons Bartran R: Psiquiatría Forense. Ed. Salvat. Barcelona, 1990. 41.

8. Skrapec C: Los motivos del asesino en serie. En Raine A; Sanmartín J: Violencia y psicopatía. Ed. Ariel. Barcelona, 2000.155-180.

9. Carrasco Gómez J]; Maza Martín JM: Manual de Psiquiatría Legal y Forense. Ed. La Ley-Actualidad. Madrid, 1997. III.4I-I8.

10. Delgado Bueno S, Esbec Rodríguez E: Imputabilidad: Concepto y perspectivas. La imputabilidad de los trastornos mentales. En Delgado Bueno S; Esbec Rodríguez E; Rodríquez Pulido F; González Rivera y Revuelta JL: Psiquiatría Legal y Foresne. Ed. Colex. Madrid, 1994. 328-329.

II. Muñoz Conde F: La imputabilidad del enfermo mental. Psicopatología. 1982; 2, 2:127-134.
12. Millon T: Trastornos de la personalidad. Mas allá del DSM IV. Ed Masson. Barcelona, 2000. 89.

13. Ortega-Monasterio L: Psicopatología jurídica y forense. Ed. Promociones y Publicaciones Universitarias. Barcelona. 1991.

14. Raine A; Sanmartín J: Violencia y psicopatía. Ed. Ariel. Barcelona, 2000. 9.

15. Hare RD: La naturaleza del psicópata: Algunas observaciones para entender la violencia depredadora humana. En Raine A; Sanmartín J: Violencia y psicopatía. Ed. Ariel. Barcelona, 2000.15-37.

16. APA: DSM IV. Manual diagnóstico y estadístico de los trastornos mentales. Ed. Masson. Barcelona, 1995.

17. Millon T: Trastornos de la personalidad. Mas allá del DSM IV. Ed Masson. Barcelona, 2000. 35.

18. Millon T: Trastornos de la personalidad. Mas allá del DSM IV. Ed Masson. Barcelona, 2000. 35.

19. Mischel W: On the interface of cognition and personality: Bellone the person-situacion debate. American Psychologist, 34, 740-754.

20. Millon T: Trastornos de la personalidad. Mas allá del DSM IV. Ed Masson. Barcelona, 2000. 36.

2I. Thetford WN, Wals R: Teorías de la personalidad y psicopatología: escuelas derivadas de la psicología y la filosofía. En Kaplan HI, Sadock BJ. Tratado de Psiquiatría. $2^{\mathrm{a}}$ ed. Reimpresión. MassonSalvat. 1992. 469-70.

22. Marcó Ribé J; Martí Tusquets JL; Pons Bartran R: Psiquiatría Forense. Ed. Salvat. Barcelona, 1990. 28.

23. Irastorza Eguskiza LI: Pluralismo mental y trastorno de la personalidad. I Congreso Virtual de Psiquiatría. I-I5 fde febrero de 2000. Conferencia 53-CI-C: Disponible en: www.psiquiatria.com/congreso/mesa53/conferencias/53_ci_c.htm. 